\title{
ENTRE MONDANITÉ ET LIBERTINAGE : FIGURES DE L'AMITIÉ DANS LES ROMANS DE CHARLES SOREL
}

Comme l'a montré Alain Génétiot, la société mondaine a « vocation à vivre, selon un idéal d'honnêteté, une vie tout à la fois contemplative et sociable, réalisée dans le cercle amical de cette petite société choisie ${ }^{1}$. Le monde est un milieu sélectif qui rassemble aristocratie de naissance et aristocratie de l'esprit, cette dernière constituant un véritable sésame pour entrer dans un groupe essentiellement coopté, où il convient d'abord de savoir se comporter et de savoir converser. Le milieu ainsi constitué est donc mélangé et plus ouvert que ne le laisserait penser une attention trop grande portée aux origines sociales. L'amitié, fondée sur une recherche commune de cet art de vivre, constitue un ferment essentiel dans l'avènement de cette sociabilité nouvelle des honnêtes gens. «[Elle] apparaît [...] comme le lien social par excellence : non le monopole d'un face à face, mais les multiples réciprocités dans lesquelles les sujets sont engagés, s'ils participent d'une même communauté $»^{2}$. Recueillant l'héritage antique, notamment celui de l'Éthique à Nicomaque, ce sentiment s'enrichit de nouvelles connotations et le mot y gagne une large polysémie au XVII ${ }^{\mathrm{e}}$ siècle. La passion amoureuse en prend parfois les détours (que l'on songe à la carte du Tendre) mais l'amitié peut aussi exprimer l'affection filiale ou bien le lien qui unit la communauté des hommes à la présence divine ${ }^{3}$ et même, dans une acception plus restreinte, le penchant

${ }^{1}$ A. Génétiot, Poétique du loisir mondain, de Voiture à La Fontaine, Champion, Paris 1997, p. 115.

${ }^{2}$ E. Méchoulan, «Amitié et générosité dans l'Astrée d'Honoré d'Urfé et Francion de Charles Sorel », Tangence 66, 2001, p. 22.

${ }^{3}$ Voir H. Merlin, «L'amitié entre le même et l'autre ou quand l'hétérogène devient principe constitutif de société », XVII siècle, $\mathrm{n}^{\circ} 205$, octobre-décembre $1999,57^{\mathrm{e}}$ année, nº 4, pp. 657-678. 
qu'on peut éprouver pour un art, ou la sympathie qui unit l'aimant et le fer. On peut également, selon Richelet, prendre de l'amitié pour un mot $^{4}$. Pareille richesse sémantique permet l'exploration d'une véritable casuistique de l'amitié dont les moralistes ont fait un sujet de prédilection en en proposant plusieurs définitions qui, comme l'a fait remarquer E. Méchoulan, jouent de leurs contradictions ${ }^{5}$.

Si l'amitié est ce lien électif de la mondanité qui refuse aux esprits trop rustres le droit d'entrée dans ce cercle restreint, on peut légitimement se demander quelle est sa place dans les romans comiques qui prétendent, eux, dépeindre la société dans son ensemble en prenant tous ses aspects en compte, y compris les plus triviaux. La « naïveté », au sens de peinture ressemblante qu'a ce mot au XVII ${ }^{\mathrm{e}}$ siècle, décrit ainsi des situations et des personnages qui laissent a priori peu de place à l'évocation de l'amitié entendue dans cette acception. Aux paysans et aux bourgeois, aux voleurs et aux maquerelles du roman comique semble dénié le droit à l'amitié, tandis qu'elle est aussi naturelle que l'air qu'ils respirent aux bergers du roman pastoral ou aux amants du roman héroïque. Mais, outre que le roman comique en général est amené à s'en prendre aux ressorts qui constituent cet univers idéalisé s'il veut s'en moquer, chez Sorel (1602?-1674), il a la particularité d'offrir une trame qui fait de l'amitié elle-même le moteur de la narration. C'est ainsi au gré des liens amicaux tissés entre les personnages principaux que se développent les aventures qui vont permettre de découvrir la société tout entière dans les trois romans comiques de Sorel.

De fait, c'est bien l'amitié de Francion pour Raymond qui forme le fil conducteur du texte : c'est chez lui que Francion découvre le portrait de Naïs, dont il tombe amoureux et dont il essaiera de retrouver le modèle en Italie. Et c'est Raymond qui l'invite à raconter ses aventures ${ }^{6}$, explicitant d'emblée que leur amitié se nourrit de leurs qualités d'esprit. C'est également parce qu'Anselme trouve des amis prêts à s'amuser de la naïveté de Louis ${ }^{7}$ et à l'entretenir dans sa folie que Le Berger extravagant $^{8}$ peut se développer sur plusieurs milliers de pages, contrecarrant le désir d'Adrian, cousin de Louis peu enclin à la plaisanterie désireux de le ramener aux réalités bourgeoises du commerce. Enfin, c'est l'amitié entre Polyandre et Néophile,

${ }^{4}$ Pour un aperçu plus détaillé des différents sens du mot, on se reportera aux dictionnaires de l'époque ainsi qu'à l'entrée « amitié » du Dictionnaire du Grand Siècle, sous la dir. de F. Bluche, Fayard, Paris 1990 .

${ }^{5}$ Voir E. Méchoulan, « Le métier d'ami », XVII siècle..., pp. 633-656.

${ }^{6}$ Cf. Ch. Sorel, Histoire comique de Francion, F. Gavarini (éd.), Folio, Paris 1996, désormais abrégé en $F R$. «Comment, Monseigneur, dit le seigneur bourguignon, est-ce ainsi que vous me privez cruellement du récit de vos plaisantes aventures ? Ignorez-vous que ces actions basses sont infiniment agréables et que nous prenons même du contentement à ouïr celles des gueux et des faquins, comme de Guzman d'Alfarache et de Lazaril de Tormes ? Comment n'en recevrai-je point à ouïr celles d'un gentilhomme écolier qui fit paraître la subtilité de son esprit et la grandeur de son courage dès sa jeunesse ? » (FR, p. 183).

${ }^{7}$ Qui s'est lui-même rebaptisé Lysis pour répondre aux canons du roman pastoral.

${ }^{8} \mathrm{Ch}$. Sorel, Le Berger extravagant. Toutes les références renvoient à l'édition d'Hervé Béchade, Slatkine Reprints, Genève 1972. Le titre sera désormais abrégé en $B E$. Nous modernisons l'orthographe. 
exprimée dès le début du roman ${ }^{9}$, qui donne à Polyandre la possibilité de séjourner à Paris durant la foire Saint-Germain et d'y croiser toutes sortes de personnages plus hauts en couleurs les uns que les autres. Si l'amitié ne constitue pas l'objet même du récit, elle en surplombe la possibilité par une complicité entre les principaux personnages, qui se reconnaissent entre honnêtes gens aptes à goûter les joies de l'esprit. Quand Néophile invite Polyandre à loger chez lui, il invoque le désir de s'entretenir en liberté avec lui, un « je-ne-sais-quoi en sa physionomie qui lui donn[e] envie de le connaître ", " une secrète sympathie », avant de conclure qu' « il ne souhaitait principalement son amitié, que pour ce qu'il lui semblait être de bonne compagnie, $\&$ c'était ce qu'il aimait selon la portée de son esprit $»^{10}$.

Le rôle de l'esprit dans la relation amicale est central. Au point que Francion, dans le récit de ses aventures à Raymond, évoque l'époque où il était au service de Clérante :

Il m'offrit un appointement honnête, que j'acceptai, pourvu que j'eusse toujours ma franchise et qu'encore que je lui rendisse des services que malaisément pusse-t-il (sic) espérer d'un autre, je n'eusse point la qualité de serviteur; il me promit qu'il ne me tiendrait jamais que comme son amil ${ }^{11}$.

Ici, la différence de condition (rappelons que Francion est d'origine noble mais pauvre) n'empêche pas une amitié que seul l'esprit de Francion a su gagner en éclairant Clérante sur des vers qui se moquaient de son ignorance :

A n'en point mentir, il avait auparavant un peu haï les lettres, et même avait blâmé quelques personnes qui s'y adonnaient, ne croyant pas que ce dût être l'occupation d'un homme noble ; mais je lui avais ôté cette imagination-là en lui remontrant doucement que ceux qui veulent commander aux autres doivent avoir plus d'esprit, non pas plus de force ainsi qu'entre les bêtes brutes ${ }^{12}$.

Avoir de l'esprit forme donc un signe de reconnaissance sur lequel s'ancre l'amitié, quand bien même cette dernière présenterait l'image peu reluisante d'un lien renforcé autour d'un bouc émissaire, comme c'est le cas dans le Berger extravagant. Les amis d'Anselme, Clarimond, Hircan et quelques autres, se mettent en effet d'accord pour s'en prendre à Lysis et s'obligent même à « lire des romans pour se rendre savants en sa doctrine [i.e. la doctrine de Lysis en matière de roman pastoral], \& tirer plus de plaisir de lui $»^{13}$. Sorel met ici en pratique la critique du roman pastoral en s'attaquant, dans le fil même de sa narration, à la noblesse du sentiment de ceux qui portent pourtant sa parole en dénonçant les excès du genre. Il ouvre ainsi une brèche dans l'image de l'amitié, que le lecteur est alors invité à interroger. Bien avant La Fontaine dans la fable des Deux amis, il pose la question : « que t'en semble, Lecteur?».

${ }^{9}$ Voir Ch. Sorel, Polyandre. Histoire comique, édition critique de P. Dandrey et C. Toublet, Klincksieck, Paris 2010, p. 45. Le titre sera désormais abrégé en Pol. Nous modernisons l'orthographe.

10 Pol, p. 46.

11 FR, p. 296.

12 Ibidem.

${ }^{13} B E$, p. $156 / 549$. 
Aussi lui emboiterons-nous le pas en examinant les différents visages de l'amitié dans les trois romans comiques de Sorel. À la récurrence des tournures prises dans un pur formalisme, s'ajoute une diversité des situations permettant d'approcher différents sens de l'amitié. L'esthétique de la varietas confère ainsi au roman comique un effet de réalisme propre à combattre une représentation stéréotypée et idéalisée de l'amitié, telle qu'elle apparaît dans les romans pastoraux et héroïques. Mais, parce qu'elle participe de cette diversité donnant l'illusion du monde comme il va, l'amitié se refuse à une définition stable et univoque. Mise à distance par le burlesque, elle manifeste une certaine ambiguïté en s'incarnant parfois dans des personnages empreints de duplicité. Elle peut alors, dans le Francion, s'infléchir vers une définition reconfigurée à l'aune de valeurs libertines qui, loin de renoncer à toute forme d'idéal, se libère du carcan des artifices sociaux imposés par le contrôle de soi cher aux honnêtes gens, pour faire l'apologie de la jouissance au sein d'une micro-société d'individus affranchis de la morale chrétienne. Reste que la chronologie de la publication de ces trois romans nous interdit d'y voir le cheminement vers une forme de plus en plus affirmée de libertinage, interprétable comme une libération progressive des chaines de la morale. La dernière édition du Francion (1633) s'accompagne en effet de remaniements qui font place à la voix de l'éditeur comme garant d'une morale préservée tout au long du texte ${ }^{14}$ et le Polyandre, dernier en date des trois textes (1648), ne reprend pas à son compte les audaces du premier Francion (1623) mais offre au contraire une vision beaucoup plus conforme à l'idée communément répandue de l'amitié, faite de loyauté et d'attention envers l'autre qui fonderaient une «éthique de la communication ${ }^{15}$.

\section{AMITIÉ ET CIVILITÉ FORMELLE}

Une lecture rapide des romans de Sorel suffit à repérer les occurrences régulières, nombreuses sans être pour autant pléthoriques eu égard au nombre de pages formé par ce corpus, du mot « ami » dans des segments phrastiques où son sens reste non marqué. En voici quelques exemples, dans lesquels on ne saurait surinvestir le sens de connotations précises :

Ha ! mon ami, reprit Lysis [qui s'adresse à Anselme pour lui expliquer qu'il craint les feux de l'amour], mon feu est un feu grégeois ${ }^{16}$.

${ }^{14}$ Sur la stratégie de l'alternance des voix narratives dans le Francion, voir M. Rosellini et G. Salvan, Le Francion de Charles Sorel, Atlande, Neuilly 2000, et l'introduction d'Anne-Elisabeth Spica à son édition de L'Anti-Roman ou l'Histoire du Berger Lysis, accompagnée de ses Remarques, seconde édition du Berger extravagant revue et augmentée par l'auteur, Champion, Paris 2014.

${ }^{15}$ L'expression est d'E. Méchoulan à propos de l'amitié chez Malebranche. $C f$. E. Méchoulan, « Le métier... », p. 655.

${ }^{16}$ BE, p. $91 / 288$. 
Il est vrai, mes amis, que je serai fort aise de me baigner tant pour me rafraichir un peu en cette saison, qui est encore bien chaude que pour me nettoyer le corps [dit Lysis à Clarimond, Philiris et Carmelin $]^{17}$.

J'envoyai le laquai d'un mien ami avec le petit paquet de papiers à la main lui [à une jeune femme qu'il veut séduire] demander si son père n'était pas à la maison ${ }^{18}$.

Un jour, j'allai voir un de mes amis, que je ne trouvai pas dans sa chambre. Il n'y avait qu'un de nos amis communs et un de ses parents, qui ne me [i.e. Francion] connaissait point ${ }^{19}$.

Or c'était un de ses amis [i.e. de Théophraste] qui s'était logé là en chambre garnie pour un jour ou deux ${ }^{20}$.

De quelle profession êtes-vous? mon bon $\mathrm{ami}^{21}$.

Tous ces exemples relèvent d'un formalisme hyperbolique se rattachant aux bienséances. Ils sont à mettre sur le même plan que les « Monsieur » dont s'apostrophent parfois les personnages et qui correspondent à un titre de simple civilité $^{22}$. La dimension affective de l'amitié y est reléguée au second plan. Marque de familiarité selon Furetière, il peut s'y mêler de la hauteur si l'on s'adresse à un inférieur, comme pourrait le laisser penser le tout dernier exemple, dans lequel les paroles sont prononcées par une mère s'adressant à celui qui courtise sa fille et qu'elle ne tient pas en estime particulière, faute de connaître sa situation sociale. De valeur axiologiquement faible, ces occurrences sont concurrencées par d'autres vocatifs, plus clairement orientés. Ainsi du " gentil berger » que Lysis adresse à Anselme au début du roman, qui a valeur laudative dans la bouche du berger extravagant.

Sorel ne s'attarde pas sur ces formules de politesse qui constituent 1'ordinaire de la civilité quotidienne. Il souligne à l'occasion combien l'usure rend ces termes creux et dénonce sur un mode plaisant la vacuité de ces tournures dans le Francion. Il qualifie ainsi de « simagrées » et de « niaiseries » ${ }^{23}$ les salamalecs des galants dont il rapporte les propos :

Je vous le dis en ami, ce n'est pas pour vous dépriser : je sais bien que vous avez assez d'autres rares vertus, car vous avez des bottes les mieux faites du monde [...] - Le plus cher de tous mes amis, lui dit l'autre en le baisant à la joue, vous me donnez des louanges que vous méritez mieux que moi! ${ }^{24}$

Au-delà de cet usage usé sur lequel il n'est pas utile d'insister, Sorel dépeint l'amitié sous des figures variées aux significations multiples.

\footnotetext{
${ }^{17} B E$, p. $352 / 360$.

18 FR, p. 270.

19 FR, p. 560.

${ }^{20}$ Pol, p. 256.

${ }^{21}$ Pol, p. 308.
}

${ }^{22}$ D'après Gaston Cayrou, ce titre implique en général considération et respect et tend à se vulgariser. Voir G. Cayrou, Dictionnaire du Français classique. La Langue du XVII siècle, Klincksieck, Paris 2000.

${ }^{23}$ FR, p. 291.

${ }^{24}$ FR, p. 290. 


\section{SENS MULTIPLES ET VARIETAS}

Sans jamais prétendre à un examen réglé des différents cas de figure, Sorel convoque au gré de la narration divers exemples illustrant l'amitié.

Il n'ignore pas la connotation amoureuse qui peut s'attacher à ce mot. Ainsi, dans sa version atténuée et familière, «m'amie » apparait dans une scène de pure farce qu'invente Clérante : il se fait passer pour un tonnelier craignant les injures de sa femme, raison pour laquelle il l'a enfermée dans un tonneau. « Baisez-moi par le trou, m'amie, lui dis-je, puis nous ferons la paix $»^{25}$.

Mais on peut aussi rencontrer une quasi synonymie entre ami et amant sans qu'aucune dimension comique n'en infléchisse le sens :

Cette courtoisie, accompagnée de mes cajoleries, la gagna facilement, si bien que je fis d'elle à mon plaisir sans qu'il m'en coûtât beaucoup de choses. Véritablement elle était fort gentille, mais depuis que je connus qu'elle n'était pas contente d'un seul ami et qu'elle commençait à se laisser aller à d'autres qu'à moi, je ne fis plus d'estime d'elle $[\ldots]^{26}$.

Les variations sur le sens qui rapprochent amour et amitié donnent lieu dans le Polyandre à une saynète badine au cours de laquelle Hypéride et Phronyme se moquent d'Orilan, qui incarne l'amoureux universel. Amour et amitié sont renvoyés dos à dos par les deux femmes qui font mine de se disputer, les exigences de l'amour empêchant de satisfaire celles de l'amitié 27 .

L'amitié, dans sa proximité avec l'amour, se présente donc comme un sentiment dont la plasticité prête à rire, tout comme dans cet épisode où la mère d'Aurélie et de Phronyme, Ragonde, prude femme venue chasser les importuns qui courtisent sa fille, se fait berner par Polyandre, déguisé en homme pieux pour moquer sa bigoterie et aussitôt adopté comme " ami » par Ragonde, selon un schéma que reprendra Tartuffe ${ }^{28}$. L'amitié, rempart contre les désordres provoqués par l'amour, est agréablement malmenée dans un comique de situation qui la désacralise plaisamment. Pour autant, s'il convient de ne pas l'idéaliser aveuglément, un juste reflet de la réalité montrera l'amitié sous un jour positif et comme l'expression d'un sentiment véritablement noble, faisant jouer le principe de la varietas, à plein.

Ainsi elle est notamment identifiée à la loyauté et à la bienveillance. Dans cette perspective, l'amitié ne saurait se soumettre à l'amour ni jouer des ambiguïtés liées à leur proximité. Polyandre et Néophile, que l'amitié a réunis

${ }^{25}$ FR, p. 338. On peut également trouver des occurrences de « m'amie » familières mais sans connotations amoureuses, comme par exemple quand Léonor s'adresse à sa servante Catherine (rebaptisée Charite par Lysis) ; BE, p. 89/279.

${ }^{26}$ FR, p. 321.

27 Pol, p. 281.

${ }^{28}$ Cf. Pol, p. 297 et sq. 
parce que c'était eux ${ }^{29}$, s'éprennent tous deux d'Aurélie. Polyandre, qui sert d'intermédiaire à Néophile auprès d'elle, se refuse à trahir son amitié et ne dit rien qu'à l'avantage de celui-ci ${ }^{30}$, selon un topos romanesque qu'exploitera peu ou prou Madame de La Fayette dans La Princesse de Montpensier. Mais là où Madame de La Fayette interrogera la lutte intérieure qui torture le personnage de Chabannes pour lui donner des accents tragiques et augustiniens, Sorel transforme la tragédie en comédie. Il imagine en effet que le père de Néophile détourne son fils d'Aurélie pour prendre sa place, demandant à Polyandre comme à un ami, de l'assister dans cette entreprise ! Et Polyandre obéit aux règles de l'amitié, se montrant complaisant envers Aesculan dans un mouvement qui l'emporte sur ses sentiments personnels ${ }^{31}$.

Outre cette démonstration de loyauté qui illustre la noblesse d'un lien amical résistant à toute épreuve, la bienveillance constitue aussi une composante valorisante de l'amitié. On le vérifie notamment dans Le Berger extravagant. Alors que les amis d'Anselme poussent le raffinement dans la moquerie jusqu'à se cultiver plus avant pour mieux entrer dans la folie de Lysis, renforçant ainsi le lien amical entre gens d'esprit qui les unit au détriment du berger mauvais lecteur de L'Astrée, l'un d'entre eux, Clarimond, va prendre ses distances avec le groupe. Il annonce à Lysis à la fin du dixième livre, et donc après s'être longuement gaussé de lui, qu'il est «plus volontiers [son] ami qu'[il] ne le pens[e] $»^{32}$. Cette annonce est suivie d'effet au quatorzième et dernier livre du roman. Clarimond demande à ses amis de mettre fin à la plaisanterie en faisant valoir qu' « il y a de la conscience à entretenir toujours un homme dedans ses extravagances qui répugne à la vraie raison $»^{33}$. Il se heurte à leur résistance avant de l'emporter par un dernier argument : « Songez qu'il n'y aura pas moins de plaisir à rendre Lysis sage, qu'à le rendre insensé comme vous désirez faire $»^{34}$.

Ce retournement en appelle à une vertu qui transcende les valeurs mondaines de l'amitié et renverse le divertissement en une action qui rapproche de Dieu par la charité ${ }^{35}$. Au moment même où Clarimond veut montrer à Lysis qu'il est « véritablement [son] ami $»^{36}$, il invoque la foi qui permet d'admirer le Créateur, et non plus la créature :

${ }^{29}$ " Ce que vous êtes suffit à vous faire aimer », affirme Polyandre à Néophile alors qu'il le conseille dans ses amours, exprimant ainsi la vraie valeur de son ami.

${ }^{30}$ Pol, p. 262 et sq.

${ }^{31} \mathrm{Cf}$. Pol, p. 271 et sq. Sur l'importance de la complaisance dans l'amitié, voir U. Langer, " De l'amitié à la complaisance : réflexion autour d'une "conversation" de Madeleine de Scudéry ", XVII siècle ..., pp. 679-686.

32 BE, p. $407 / 508$.

33 BE, p. $527 / 163$.

34 BE, p. $528 / 165$.

${ }^{35}$ Les effets de l'honnête amitié sont étudiés par E. Méchoulan dans «Amitié et générosité ... », p. 25 et sq.

36 BE, p. 530/173. 
Vous avez parlé de votre maitresse comme d'une chose divine, encore que vous puissiez bien savoir que c'est une fille mortelle, qui boit et mange comme vous. Vous avez dit que vous n'aviez de la foi que pour elle, avez-vous donc oublié la foi de vos pères pour être un idolâtre? ${ }^{37}$

L'amitié véritable rachète les excès de l'amitié mondaine. Les valeurs de l'esprit sont transcendées par celles de la foi et la quête amoureuse de Lysis se mue en apologie de la religion chrétienne. La belle Charite (c'est le nom choisi par Lysis pour désigner la servante Catherine dont il est amoureux) se métamorphose en vertu théologale. L'interprétation de cette fin édifiante reste problématique. Outre que la bienveillance charitable de Clarimond est longue à se manifester, le roman a offert des épisodes parfois cruels envers Lysis ou son serviteur Carmelin ${ }^{38}$. On peut se demander si le retournement final ne satisfait pas d'abord aux exigences de fin heureuse, constituant un artifice efficace pour sceller le sort de Lysis et son retour à la raison. D'autant qu'au dénouement, Clarimond présente sa vie et celle de ses amis comme une sorte d'idéal à atteindre pour Lysis :

L'on peut bien vivre aux champs sans porter une houlette. Voyez comme est Hircan, voyez comme est Oronte, \& comme je suis. Nous demeurons toujours ici ; nous chassons, nous pêchons, nous nous promenons, nous faisons tout ce qui nous plaît ; n'est-ce pas tout ce que vous désirez? ${ }^{39}$

On peut voir dans cette évocation comme l'idéal d'une certaine aristocratie épicurienne, auquel il manque néanmoins un divertissement, pratiqué par le groupe d'amis tout au long du roman : la lecture. Après les discours innombrables auxquels ce passe-temps a donné lieu au fil du récit, on imagine mal les personnages s'en passer et se priver ainsi d'un des plaisirs de l'amitié, celui de la conversation nourrie à cette culture commune. La charité dans l'amitié est-elle compatible avec l'esprit de raillerie qui unit les esprits dans la distance désinvolte qui fait le sel de la conversation ? Le roman comique, qui repose sur la complicité avec le lecteur, peut-il, comme semble vouloir le faire Le Berger extravagant, proposer autre chose qu'une peinture de l'amitié bigarrée aux couleurs de la varietas et laissée à la libre appréciation du lecteur?

Sorel ne procède pas à une rédemption en bonne et due forme de l'amitié par la charité. Bien avant de la convoquer, il s'est appliqué à rendre ses personnages complexes. On l'a vu avec Clarimond, qui prend bien du plaisir à se moquer de Lysis avant de se déclarer son ami. Le naïf berger lui-même n'est pas irréprochable dans les relations qu'il entretient avec Carmelin. Dans un premier temps, il est touché par son esprit et le prend à son service. C'est ainsi qu'il le présente à Clarimond comme un « honnête homme $»^{40}$. Mais il le malmène parfois durement, comme un valet et non comme un ami. Il le traite de "gros âne ${ }^{41}$ et considère

${ }^{37}$ BE, p. $530 / 174$.

${ }^{38}$ Notamment lorsque Carmelin est fouetté par la bande d'amis au cours de l'épisode de la métamorphose en arbre.

${ }^{39}$ BE, p. 539/209.

${ }^{40} B E$, p. $539 / 209$.

${ }^{41}$ BE, p. $166 / 587$. 
qu'il n'est guère capable que de raconter des bouffonneries ${ }^{42}$, il admet l'avoir traité rudement tout en cherchant à s'en dédouaner ${ }^{43}$. Pourtant, quand il ne le trouve pas, il l'appelle : «Carmelin, Carmelin, où es-tu ? mon ami, mon mignon, mes délices, mes amourettes $\rangle^{44}$. Il répond à la demande de son valet qui aspire à un autre statut en le promouvant « commis en fait de Bergerie » car « [il] l'estime bien plus digne d'être berger qu'[il] ne fais[ait jusque-là] ${ }^{45}$. Outre que cette complexité des relations entre les personnages participe de l'illusion réaliste en évitant des stéréotypes trop caricaturaux et donne des couleurs variées au tableau, elle montre qu'en matière d'amitié, les comportements s'adaptent en fonction des registres, suggérant clairement que l'amitié n'est pas de même nature selon qu'on appartient à tel ou tel milieu, et selon que le roman transpose la relation amicale dans tel ou tel registre littéraire. Personnages éminemment comiques, Lysis et Carmelin entretiennent une amitié qui prend nécessairement un tour burlesque et si Lysis voit d'abord en Carmelin un honnête homme, son regard ne saurait se maintenir à pareille hauteur. La condition de valet de Carmelin entraine une dégradation de l'amitié que lui porte Lysis, dont l'affection est pourtant réelle.

Sujette à ces variations tant thématiques que de registre, l'amitié n'offre pas d'image stable qui permette de la définir clairement. Elle apparaît bien comme un sentiment déterminant du lien social, allant au-delà de la simple civilité pour interroger la nature même de ce qui unit les hommes, entre eux et au divin. Mais elle ne se présente pas pour autant comme un pur idéal à atteindre dont les contenus seraient nettement définis. Sorel s'est pourtant évertué dans son premier roman comique, le Francion, à trouver la possibilité d'un lien capable de rassembler les individus selon un autre modèle.

\section{LIBERTINAGE ET AMITIÉ}

Des trois romans comiques de Sorel, le Francion est celui où l'amitié est la plus explicitement commentée. Que ce soit pour souligner qu'elle ne saurait s'épanouir qu'entre égaux (par exemple entre Francion et Clérante, tous deux d'humeur « franche ${ }^{46}$ ) ou pour affirmer qu'avoir beaucoup d'amis est « le plus grand plaisir de la vie ${ }^{47}$, au point que le pseudo-éditeur du Francion « aime mieux perdre [ses] bons mots que [ses] amis $»^{48}$, les pages où elle est mentionnée sont nombreuses. C'est aussi le roman dont la critique s'est le plus volontiers emparé pour tenter de

\footnotetext{
${ }^{42} C f . B E$, p. $320 / 232$ et sq.

43 Cf. BE, p. 416/616.

${ }^{44} B E$, p. 222/811. Il l'appelle « mon ami » à plusieurs reprises, par ex. p.184/667, p. 210/762.

${ }^{45}$ BE, p. 251/924-925.

${ }^{46}$ FR, p. 332.

${ }^{47}$ FR, p. 448

${ }^{48}$ FR, p. 384
} 
cerner comment s'y jouait l'inscription du libertinage. Il est désormais admis que la multiplicité des voix narratives, y compris celle du pseudo-éditeur dans la réédition de 1633, participe d'une stratégie de dissimulation/monstration des valeurs libertines $^{49}$. Au fondement de ces valeurs se trouve l'amitié. Méchoulan a montré comment Sorel s'inscrivait dans une pensée politique de l'amitié : Francion « met sur le même plan politique, amour et fortune [...]. Mais c'est pour mieux en déchiffrer les ressorts et en maîtriser les effets ${ }^{50}$. S'appuyant sur les valeurs de la noblesse, l'amitié développe dans le texte, selon lui, une « stratégie de clans et d'affrontements » qui enserre la morale dans « le filet d'une économie politique $»^{51}$. Le sexe y apparaît comme une force libératrice loin de la pure débauche. « Il devient une activité où se reconnaissent des êtres à part qui allient, sous le signe d'une société d'amis (et d'amies), l'élégance du langage au raffinement des gestes $»^{52}$.

On peut pourtant se demander si cette société d'amis parvient véritablement à ses fins. La compagnie des Braves et Généreux que forment Francion et ses compères au livre VI rassemble des « personnes toutes braves et ennemies de la sottise et de l'ignorance, pour converser ensemble et faire une infinité de gentillesses $»^{53}$. Si les grands seigneurs recherchent leur amitié, il n'est jamais fait mention explicite d'un lien amical les unissant et, très rapidement, la compagnie s'essouffle. Est-ce faute d'amitié réelle?

De fait, au moment même où le coup d'arrêt de la bande se profile, se manifeste une impatience de Francion à l'égard des Braves et Généreux peu compatible avec un lien amical ${ }^{54}$. Bien plus, cette impatience se transforme en égoïsme ${ }^{55}$ et laisse bientôt apparaître une véritable duplicité du personnage :

De mes anciens camarades, il n'y en avait plus que deux ou trois de qui je fisse état ; pour les autres, que j'avais fait semblant de chérir à cause du profit que j'en tirais (ce qui est une invention dont l'on se peut quelquefois servir sans devoir craindre un juste blâme), je ne traitais plus avec eux si familièrement, pour leur montrer qu'ils n'étaient rien au prix de moi et qu'ils se rendaient désagréables par leurs imperfections ${ }^{56}$.

Cette duplicité revient à plusieurs reprises dans le récit. Francion n'a aucun scrupule à jouir de la femme aimée de Clérante et s'en justifie en ces termes : « Mon plaisir ne me devait-il pas toucher de plus près que celui d'un autre ? ${ }^{57}$.

\footnotetext{
${ }^{49}$ Voir notamment M. Rosellini et G. Salvan, op. cit.

${ }^{50}$ E. Méchoulan, «Amitié et générosité... », pp. 31-32.

${ }^{51}$ Ibidem, pp. 33-34.

52 Ibidem.

${ }^{53}$ FR, p. 283.

54 « Je tâchais de supporter leur humeur quand je me trouvais avec eux ; mais je ne les hantais que le moins qu'il m'était possible et me tenais fort souvent chez moi, figurant d'être mal disposé, pour éviter leur fréquentation » (FR, p. 287).

55 « Je ne songeais plus qu'à procurer le contentement de moi seul» (FR, p. 288).

${ }^{56}$ FR, p. 297.

${ }^{57}$ FR, p. 318.
} 
On rétorquera que cet individualisme n'est pas un obstacle à la constitution du petit groupe d'amis formé un peu plus loin dans le récit qui, sans se fixer de programme comme l'avait fait la bande des Généreux ${ }^{58}$, place le compagnonnage de Francion et de Raymond explicitement sous le signe d'une amitié qui comprend jusqu'au partage des amours dans la débauche ${ }^{59}$. L'amitié qui unit ces deux personnages ne se dément pas au fil du roman. Mieux, elle est relayée par une surenchère verbale, qui la sanctifie en évoquant les liens indissolubles de la fraternité, et ce, alors même qu'ils contreviennent par le projet d'une union entre Francion et Naïs à la règle autrefois formulée ensemble contre le mariage ${ }^{60}$.

$\mathrm{Au}$ point que tous deux sont mélancoliques à l'idée que ce mariage ne puisse avoir lieu et que Francion ne soit contraint de quitter Rome en y abandonnant Raymond ${ }^{61}$. L'amitié transcende alors toute marque définitoire extérieure pour s'exprimer dans le pur ressenti des affects qui ne trompent pas, parce qu'ils ne se contrôlent pas. Elle s'affiche comme indice d'une distinction sociale réservée à une élite capable d'ébranler les principes de la sociabilité dominante des honnêtes gens par l'apologie de la jouissance et de l'individualisme, qui contreviennent à l'idée d'une amitié mondaine faite de mesure et de contrôle de soi. Les humeurs de la mélancolie révèlent l'amitié libertine dans la transparence d'une complexion qui dirait la vérité de l'homme au-delà des artifices de la sociabilité.

L'amitié dans les romans de Charles Sorel se donne ainsi à voir dans la complexité du travail littéraire. Prise au piège du roman comique, elle s'offre à la bigarrure d'une peinture naïve qui, par nécessité générique, s'en prend à une définition restreinte de la notion en se jouant des stéréotypes et des registres. Sorel offre donc des images variées de l'amitié dans ses différents romans sans se soucier de procéder à une étude raisonnée des cas, comme le feraient plus volontiers les moralistes. Il ouvre un dialogue de romancier à romancier en opposant le genre comique au genre pastoral. Ainsi se trouve dépassée l'apparente contradiction entre une amitié qui trouverait son sens profond dans la charité et une amitié qui ferait au contraire fi de toute morale chrétienne pour prôner un credo libertin. Si les stratégies de dissimulation libertine invitent à penser que là se cache la pensée secrète de l'auteur, c'est pourtant en s'en tenant à son expression littéraire qu'il faut appréhender l'amitié. En ce sens, difficile de trancher. Il n'y a pas de théorie libertine globale et unifiée de l'amitié dans les romans de Sorel. Mais bien plutôt un entre-deux littéraire, entre mondanité et libertinage, qui laisse le lecteur répondre par lui-même à la question de La Fontaine.

58 Cf. FR, p. 283.

59 « Mon cher ami, c'est maintenant que je vous donnerai des témoignages de l'affection que je vous porte, vous laissant jouir de toutes les délices dont je me pourrai aviser », dit Raymond à Francion, lui annonçant derechef qu'il a fait venir cinq demoiselles, dont « son » Hélène, afin qu'il choisisse parmi elles ( $F R$, p. 388).

${ }^{60} C f$. FR, respectivement pp. 557 et 399.

${ }^{61}$ FR, p. 622. 


\title{
BETWEEN WORLDLY FRIENDSHIP AND LIBERTINAGE : FIGURES OF FRIENDSHIP IN CHARLES SOREL'S NOVELS
}

\begin{abstract}
Summary
Friendship was an essential ferment in the advent of a new kind of sociability in the seventeenth-century France. The comic novel — and especially Sorel's works — with its ambition to accurately portraiting the world, provides a unique vantage point for observing this phenomenon. Whether honest friendship is praised or mocked, Sorel offers various images of a link between the characters that is often tantamount to belonging to the same environment. As a criterion of social discrimination, friendship is shown in an ambivalent light: thus, Lysis is mocked by his friends because he does not control gallantry codes (Berger extravagant). Yet, the purpose is not to denigrate a virtue regarded as fundamental in the social life. The reason why Neophile and Polyandre are friends yet love rivals (Polyandre), just as are Francion and Cléandre (Francion), is that narrative techniques shift the painting of friendship towards an aesthetic of varietas meant to make it plausible. Thus the characters embody different variations of the stereotype of worldly friendship, allowing the reader to question its role in the society of the time. This worldly aspect is complemented by Sorel with another one in which the society of friends constitutes a crucible for other values that are capable to transcend the artifice of the most commonly shared social codes to assert a libertine credo (Francion).
\end{abstract}

Key words: Sorel, friendship, worldliness, libertinage, comic novel, varietas, ambiguity. 